\section{LEPTOSPIROSIS EN PACIENTES CON RESULTADO NEGATIVO PARA DENGUE, ZIKA Y CHIKUNGUNYA EN JAÉN, PERÚ}

\author{
LEPTOSPIROSIS IN PATIENTS WITH \\ NEGATIVE RESULTS FOR DENGUE, ZIKA, AND \\ CHIKUNGUNYA IN JAEN, PERU
}

\section{Xiommara Alvarado Dávila1,a , Edwin Yober Gavidia Olivera $^{2, b}$, Blanca Nieves Zulueta Vásquez ${ }^{1, a}$, Franco Romaní3,b}

Sr. Editor. La leptospirosis está ampliamente distribuida en Perú. En 2018, 304 distritos reportaron al menos un caso. El Centro Nacional de Epidemiología, Prevención y Control de Enfermedades reportó que en 2018 la incidencia acumulada por 100 mil habitantes llegó a 8,7 (2785 casos confirmados, de los cuales 57 fueron de Cajamarca). Jaén es una provincia de la región Cajamarca, se encuentra en un área de clima cálido semiseco caracterizado por abundantes precipitaciones durante el año, con temperatura promedio anual de $27^{\circ} \mathrm{C}$, y humedad permanente.

Presentamos la frecuencia de evidencia serológica de leptospirosis en 101 pobladores con ficha de investigación clínico epidemiológica para la vigilancia de dengue, chikungunya, zika y otras arbovirosis, captados entre el 1 de enero y 28 de febrero del 2019, y cuyas muestras resultaron negativas para dengue (ELISA IgM y ELISA antígeno NS1), zika y chikungunya (ELISA IgM). Dichos procedimientos fueron realizados en el Laboratorio Referencial de Salud Pública de Jaén. Además, las muestras fueron analizadas con ELISA IgM para leptospirosis; aquellas con resultados reactivos e indeterminados fueron remitidas al Instituto Nacional de Salud para confirmación mediante aglutinación microscópica (MAT). Los datos fueron generados por el sistema de vigilancia epidemiológica a cargo de la Dirección de Salud de Jaén, de quien obtuvimos autorización para uso y publicación de la información.

La proporción de MAT positivo fue $31,7 \%$, identificamos 14 serovares, los más frecuentes fueron Varillal $(20,8 \%)$ e Icterohaemorrhagiae $(19,8 \%)$ (Tabla 1). Entre los MAT

\footnotetext{
Laboratorio Referencial de Salud Pública, Dirección Subregional de Salud I Jaén. Cajamarca, Perú.

2 Dirección Subregional de Salud I Jaén. Cajamarca, Perú.

3 Oficina General de Investigación y Transferencia Tecnológica, Instituto Nacional. Lima, Perú.

a Bióloga; ${ }^{b}$ Médico

Recibido: 15/04/2019 Aprobado: 17/07/2019 En línea:31/07/2019
}

Citar como: Alvarado Dávila X, Gavidia Olivera EY, Zulueta Vásquez BN, Romaní F. Leptospirosis en pacientes con resultado negativo para dengue, zika y chikungunya en Jaén, Perú. Rev Peru Med Exp Salud Publica. 2019;36(3):535-6. doi: http://dx.doi.org/10.17843/rpmesp.2019.363.4457.
Tabla 1. Características demográficas, clínicas y de laboratorio de pacientes con resultados negativos para arbovirus captados en la Red de Salud de Jaén (Cajamarca) entre enero y febrero del $2019(\mathrm{~N}=101)$

\begin{tabular}{|c|c|c|}
\hline Característica & $\mathbf{n}$ & $\%$ \\
\hline Edad (años), media (desviación estándar) * & \multicolumn{2}{|c|}{$30,3(19,0)$} \\
\hline Sexo masculino & 47 & 46,5 \\
\hline \multicolumn{3}{|l|}{ Procedencia } \\
\hline Jaén & 34 & 33,7 \\
\hline Fila Alta & 22 & 21,8 \\
\hline Los Sauces & 14 & 13,9 \\
\hline Morro Solar & 11 & 10,9 \\
\hline Otras áreas & 20 & 19,8 \\
\hline \multicolumn{3}{|l|}{ Sintomatología } \\
\hline Fiebre & 88 & 87,1 \\
\hline Cefalea & 81 & 80,2 \\
\hline Mialgia & 68 & 67,3 \\
\hline Dolor ocular & 64 & 63,4 \\
\hline Artralgia & 48 & 47,5 \\
\hline Náuseas & 47 & 46,5 \\
\hline Dolor lumbar & 44 & 43,6 \\
\hline Rash & 41 & 40,6 \\
\hline \multicolumn{3}{|l|}{ ELISA IgM leptospira } \\
\hline Reactivo & 33 & 32,7 \\
\hline No reactivo & 55 & 54,5 \\
\hline Indeterminado & 13 & 12,9 \\
\hline Resultado positivo de $\mathrm{MAT}^{\dagger}$ & 32 & 31,7 \\
\hline \multicolumn{3}{|l|}{ Serovares identificados } \\
\hline Varillal & 21 & 20,8 \\
\hline Icterohaemorrhagiae & 20 & 19,8 \\
\hline Hurstbridge & 14 & 13,9 \\
\hline Hardjo & 9 & 8,9 \\
\hline Bataviae & 4 & 3,9 \\
\hline Coxi & 3 & 2,9 \\
\hline Bratislava & 2 & 1,9 \\
\hline Canicola & 2 & 1,9 \\
\hline Grippotyphosa & 2 & 1,9 \\
\hline Djasiman & 1 & 0,9 \\
\hline Cynopteri & 1 & 0,9 \\
\hline Australis & 1 & 0,9 \\
\hline Copenhageni & 1 & 0,9 \\
\hline Panama & 1 & 0,9 \\
\hline
\end{tabular}

MAT: prueba de aglutinación microscópica

* Rango de edad: 0,17 a 85 años

† Prueba de aglutinación microscópica: 24 MAT positivo (cinco fueron negativas y en cuatro no se realizó) entre los positivos al ELISA IgM. Ocho MAT positivos (tres fueron negativas y en dos no se realizó) entre indeterminados al ELISA IgM.

positivos, nueve fueron reactivos a un solo tipo de serovar, diez a dos, seis a tres, cinco a cuatro serovares, uno a siete y otro a ocho serovares. Los síntomas más frecuentes entre los positivos fueron cefalea $(87,5 \%, n=28)$, fiebre $(84,4 \%$, $n=27)$, mialgia $(75,0 \%, n=24)$, dolor ocular $(56,3 \%, n=18)$, artralgia $(53,1 \%, n=17)$, rash $(50,0 \%, n=16)$, nauseas $(43,8 \%, n=14)$ y dolor lumbar $(37,5 \%, n=12)$. 
Entre los varones, 42,6\% (20/47) fueron MAT reactivos, entre mujeres $22,2 \%$ (12/54) fueron reactivas. La proporción de reactivos incrementó según grupo etario, entre $<18$ años fue de $21,4 \%(6 / 28)$, entre 18 y 64 años de $34,3 \%$ (23/67) $y$ en $\geq 65$ años de 50,0\% (3/6). Entre los residentes de Morro Solar el 50,0\% (6/11) fueron reactivos, 35,5\% (7/22) en Fila Alta, 24,4\% (9/34) en Jaén, 15,4\% (1/14) en los Sauces, y 9 de 20 residentes de otras áreas de Jaén. Entre aquellos dedicados a la agricultura $71,4 \%$ (5/7) tuvieron MAT reactivo.

Un estudio en Jaén realizado entre mayo de 2004 y abril de 2005 , encontró que $11,1 \%$ de 1039 febriles tuvieron sólo leptospirosis, además reportó 15 serovares diferentes, los más frecuentes fueron Varillal $(35,7 \%)$, Bratislava $(32,5 \%)$ y Cynopteri $(12,7 \%)^{(1)}$, además hubo un incremento de casos en el mes de enero respecto a otras etiologías de sindrome febril.

Encontramos serovares que también han sido reportados en estudios previos. En agricultores de arroz de la región San Martín, los serovares Icterohaemorrhagiae, Autumnalis, Panama y Australis fueron los más frecuentes. Nuestro reporte ratifica la circulación de múltiples serovares en áreas endémicas: 15 serovares en Jaén en 2004-2005 (1), 19 en la región San Martin (2010) ${ }^{(2)}$, y 14 en el presente estudio. También encontramos que entre agricultores la proporción de reactividad fue superior al 64,4\% reportado en agricultores de San Martín (2).

El $13,9 \%$ de muestras fueron positivas al serovar Hurstbridge, el cual no es comúnmente reportado en estudios de seroprevalencia en humanos en Perú. Sin embargo, sí se ha reportado en reservorios mamíferos, por ejemplo, en $2,0 \%$ de 305 canes con diagnóstico presuntivo de leptospirosis de Lima ${ }^{(3)}$, y en 9,5\% de 126 alpacas de la Estación Experimental Maranganí en la provincia de Canchis (Cusco) ${ }^{(4)}$.

Entre las limitaciones: no se descartaron todas las causas de síndrome febril (malaria, rickettsiosis, oropuche, entre otras); en ocho muestras no se realizó el MAT, lo cual pudo afectar la estimación puntual calculada. Estos resultados no reflejan la magnitud de la leptospirosis en la provincia de Jaén, debido a que fue realizado en sintomáticos, y por las limitaciones en la interpretación de un MAT único. Dicho método puede indicar títulos de anticuerpos persistentemente elevados lo cual hace imposible estimar el tiempo de infección basándose en un solo título específico, y atribuir el cuadro clínico a un determinado serovar (5); por otro lado, en la fase aguda, los anticuerpos específicos pueden no ser detectados por reacciones serológicas, lo cual genera falsos negativos, que sí podrían ser identificados por técnicas moleculares como la reacción en cadena de polimerasa ${ }^{(6)}$.

En conclusión, en tres de diez casos en los cuales se descartó las principales metaxénicas virales existió confirmación de anticuerpos contra leptospira. También reportamos la circulación de múltiples serovares, incluyendo el serovar Hurstbridge, identificado previamente en mamíferos reservorios. Recomendamos evaluar la magnitud de infección asintomática en esta región, así como estudiar la circulación de los diversos serovares en reservorios, e identificar qué factores de riesgo tienen la población del área estudiada.

Contribuciones de los autores: XAD participó en la concepción del estudio, realizó el procesamiento diagnóstico para Leptospira, trabajó la base de datos. EYGO participó en la concepción del estudio y en la limpieza de la base de datos. BNZV participó en el procesamiento diagnóstico de las metaxénicas virales y en la construcción de la base de datos. FRR participó en la concepción del estudio, en la limpieza de la base de datos, análisis de datos y redacción del borrador. Todos los autores participaron en la revisión de las versiones del manuscrito, y aprobación final.

Fuentes de financiamiento: el presente estudio fue autofinanciado.

Conflicto de interés: Los autores declaran no tener conflictos de intereses. FR se desempeña como Editor Científico de la Revista Peruana de Medicina Experimental y Salud Pública.

\section{REFERENCIAS BIBLIOGRÁFICAS}

1. Troyes L, Fuentes L, Troyes M, Canelo L, García M, Anaya $\mathrm{E}$, et al. Etiología del síndrome febril agudo en la provincia de Jaén, Perú 2004-2005. Rev Peru Med Exp Salud Pública. 2006;23(1):5-11.

2. Alarcón-Villaverde JO, Romani-Romani F, Tejada RA, Wong-Chero P, Céspedes-Zambrano M. Seroprevalencia de leptospirosis y características asociadas en agricultores de arroz de una región tropical del Perú. Rev Peru Med Exp Salud Publica. 2014;31(2):195-203.

3. Siuce J, Calle S, Pinto C, Pacheco G, Salvatierra G. Identificación de serogrupos patógenos de Leptospira en canes domésticos. Rev Inv Vet Peru. 2015;26(4):664-75. doi: 10.15381/rivep.v26i4.11221.

4. Better BY, Salvatierra G, Siuce MJ, Calle ES. Identificación de serogrupos de Leptospira spp en alpacas del Centro de Investigación IVITA (Maranganí, Cusco). Rev Investig Vet Perú. 2018;29(2):619-24. doi: 10.15381/rivep.v29i2.14518.

5. Cumberland P, Everard CO, Wheeler JG, Levett PN. Persistence of anti-leptospiral IgM, IgG and agglutinating antibodies in patients presenting with acute febrile illness in Barbados 1979-1989. Eur J Epidemiol. 2001; 17(7):601-8.

6. Muller S, Assenga J, Matemba L, Misinzo G, Kazwala R. Human leptospirosis in Tanzania: sequencing and phylogenetic analysis confirm that pathogenic Leptospira species circulate among agro-pastoralists living in Katavi-Rukwa ecosystem. BMC Infect Dis. 2016;16:273. doi: 10.1186/s12879-016-1588-x.

Correspondencia: Xiommara Alvarado Dávila

Dirección: Laboratorio Referencial de Salud Pública, Dirección Subregional de Salud I Jaén. Cajamarca, Perú

Teléfono: (511) 972626238

Correo electrónico:analisis_xiomy@hotmail.com 